\title{
The influenza matrix protein 2 as a vaccine target
}

\author{
Xavier Sadens \\ D epartment for M olecular \\ Biomedical Research, VIB, \\ Ghent, Belgium, \\ and, \\ D epartment for M olecular \\ Biology, Ghent University, \\ G hent Technologi epark 927, \\ B-9052 G hent, Belgium \\ Tel.: +32 93313620 ; \\ Fax: +32 93313609 ; \\ xavier.saelens@dmbr.ugent.be
}

\begin{abstract}
Matrix protein (M)2 is an Influenza A, type III membrane protein with an extracellular domain (ectodomain of M2 (M2e)) of 23 amino acid residues, which is strongly conserved across virus strains. M2 fulfills an important biological function in the life cycle of the Influenza A virus and has been a target of antiviral drugs. M2e has generated much interest as a potential vaccine target, and a clinical M2e vaccine trial was initiated in 2007. The advantage of $\mathrm{M} 2 \mathrm{e}$ compared with hemagglutinin, the prime antigen target in conventional influenza vaccines, is that its sequence is conserved. This means that a stable, efficacious and easily produced M2e-based vaccine would provide protection not only against drifting seasonal influenza epidemic strains, but would also make it possible to vaccinate in anticipation of an emerging pandemic. Furthermore, most reported M2e-based vaccines are produced by economical and safe technologies. Ig $G$ subtype antibodies directed against M2e can prevent death from influenza and reduce morbidity in animal models for influenza disease. The immunological mechanism that mediates protection by anti-M2e antibodies is not completely understood, but it probably involves antibody-mediated cellular cytotoxicity. This review summarizes the findings on M2e vaccine candidates and addresses some of the key unanswered questions about this promising Influenza A vaccine target: what is its likely mechanism of action? Which measurable parameters correlate with protection? And what can be expected from clinical use of an M2e-based vaccine?
\end{abstract}

Prophylactic vaccination aims to induce protection against a potential future disease. One important prerequisite for successful vaccination against a microbial infection is knowledge of the antigenic nature of the pathogen. When developing vaccines against infectious agents that are antigenically invariant, or nearly so, such as Variola virus and Poliovirus, vaccine composition is well-defined and hardly changes over time. However, the dominant antigenic properties of human Influenza A and B viruses, the causative agents of influenza, vary constantly. This implies that the composition of influenza vaccines requires frequent updating, demanding considerable flexibility from the vaccine manufacturer. Changes in composition are based on global monitoring by W HO reference laboratories to identify the influenza strains that are most likely to cause the next epidemic [1]. Adaptive immunoselective pressure in the human host leads to the emergence of influenza virus escape variants that replace previously circulating strains. At the molecular level, this immune escape originates from the low fidelity of the viral RNA-dependent RNA polymerase, the segmented RNA genome and the antigenic flexibility of the viral hemagglutinin $(\mathrm{HA})$ and neuraminidase (NA), the dominant influenza virus antigens (Figure 1A). This continuous antigenic evolution of influenza virus has been coined 'antigenic drift' and 'antigenic shift', denoting subtle and major changes, respectively [2-4]. Licensed influenza vaccines, in other words, inactivated vaccines and live attenuated vaccines, are based on the antigenicity of $\mathrm{HA}$ and provide effective protection against specific, antigenically matching virus strains, but little protection against more distant strains, even those belonging to the same subtype [5], and they do not provide heterosubtypic immunity. Influenza vaccines used in most countries are based on purified components derived from inactivated virus, whereas in Russia, and more recently in the USA, live-attenuated influenza vaccines have been licensed. Vaccine seed viruses are mostly propagated in embryonated chicken eggs, but cultures in mammalian cells (e.g., MDCK or Vero cells) are being explored for production and isolation of the vaccine antigens. The production process necessitates biological containment, and the entire program to obtain sufficient doses for a vaccination campaign requires 3-6 months. Vaccination of the public with trivalent influenza vaccines (two Influenza $A$ strains and one Influenza B strain) can be $70-90 \%$ effective in pre venting influenza-induced illness in children and adults $[6,7]$. In the elderly, who have a high risk of developing severe disease and increased mortality 


\section{Figure 1. Influenza A virus particle and its matrix protein 2 proton channel.}

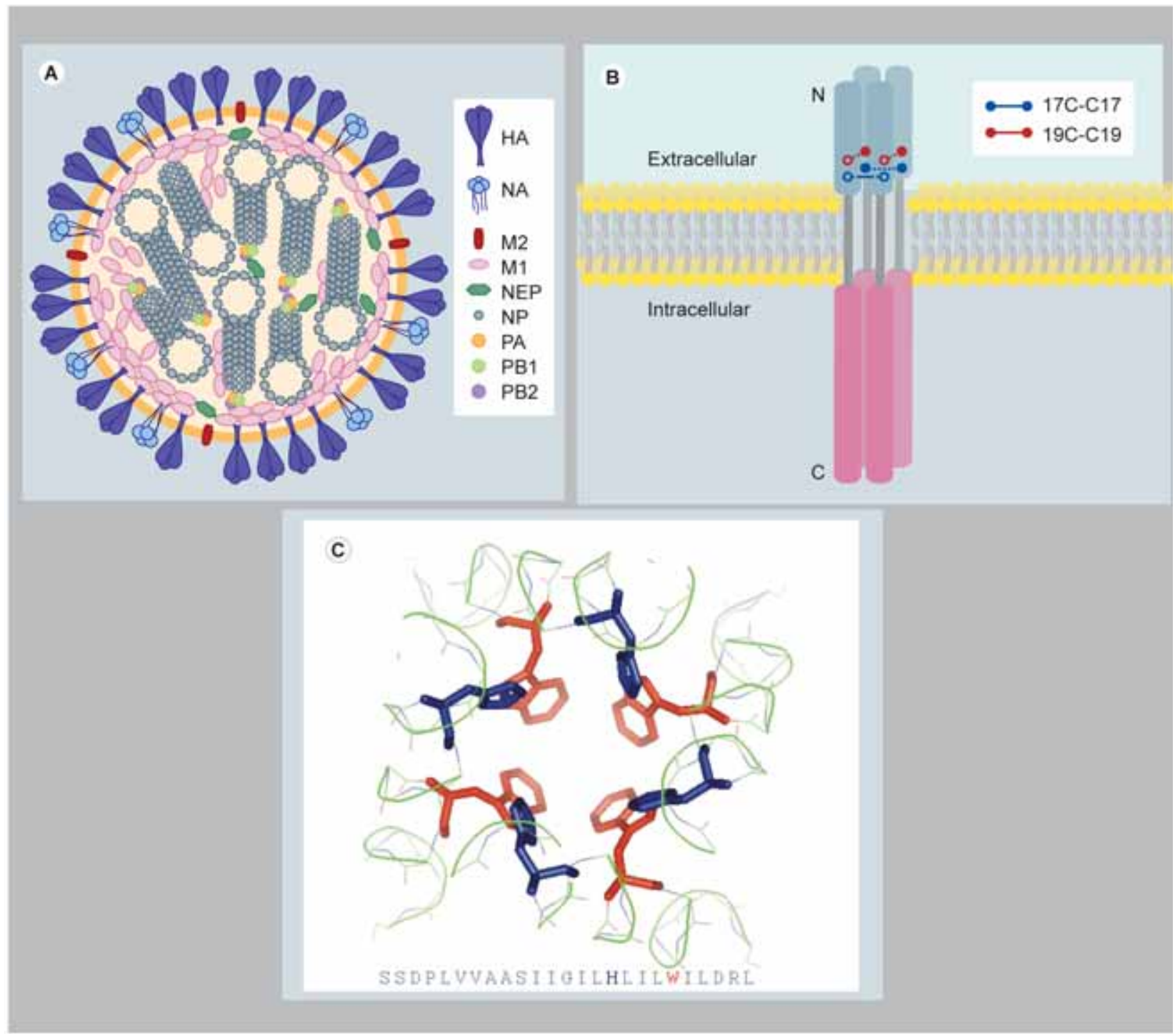

(A) Model of Influenza A virus particle. HA, NA and M 2 are embedded in the lipid membrane that surrounds the matrix virion, which is composed of M 1. The viral genome consists of eight RNA segments with negative polarity and partially complementary termini. The genomic RNA segments are wrapped around NP and its 5' and 3 'termini are associated with polymerases PB1, PB2 and PA, thereby forming a ribonucleoprotein complex. The NEP is also packaged in the virions. (B) M embrane orientation of M 2 and its oligomeric structure. M2 is a type III membrane protein with an extracellular domain (ectodomain of M2 [M 2e]) of 23 amino acid residues (aa), from which the initiator methionine is post-translationally removed, a transmembrane domain of 19 aa and an intracellular domain of 54 aa. The disulfide bridges between Cys17 and Cys19 are indicated. Extracellular denotes the exterior of a cell (e.g., infected cell), the lumen of vesicles of the eukaryotic secretion pathway (e.g., Golgi) or the exterior of an Influenza A virus particle. Intracellular denotes the cytoplasm of a cell or the virion interior. (C) View into the transmembrane domain of M2 from the extracellular site. The transmembrane domain presumably forms a tetrameric helical bundle. Individual helices are shown in green. His37 is shown in blue and Trp41 in red in the HXXXW motif. His37 and Trp41 of adjacent helices interact and control the influx of protons in a pH-dependent way. This process involves protonation of the imidazole group of His37 and electrons in the indolyl ring of Trp41. The structure was determined by solid state nuclear magnetic resonance of the peptide with the indicated sequence corresponding to the transmembrane domain of $M 2$ of $A / H o n g$ Kong/1073/99 (H9N2) [64].

Figure 1C was made using PyM OL (DeLano WL [2002]; The PyM OL M olecular Graphics System. DeLano Scientific LLC, Palo Alto, CA, USA).

HA: Hemagglutinin; M: M atrix protein; NA: Neuraminidase; NEP: Nuclear export protein; NP: Nucleoprotein; PA: Acidic polymerase; PB: Basic polymerase. 
due to influenza, effectiveness of influenza vaccines reaches only $30-50 \%$, leaving considerable room for improvement [8-10].

Influenza A viruses have intrinsic pandemic potential, mainly because of their zoonotic nature. However, out of $16 \mathrm{HA}$ and nine $\mathrm{N} \mathrm{A}$ subtypes [11], only H $1 \mathrm{~N} 1, \mathrm{H} 2 \mathrm{~N} 2, \mathrm{H} 3 \mathrm{~N} 2$ and possibly $\mathrm{H} 3 \mathrm{~N} 8$ subtypes are known to exist, or have existed, as fully human-adapted Influenza A viruses. A number of avian influenza strains, such as the highly pathogenic $\mathrm{H} 5 \mathrm{~N} 1$ strain, can occasionally cross the species barrier and infect humans, a reminder of this virus's potential for causing devastating pandemics [12]. Influenza pandemics have been documented only for A viruses. A pandemic starts when a virus with an $\mathrm{HA}$ subtype that is different from contemporary strains infects humans and spreads from person-to-person. This so-called shift of the H A subtype can be accompanied by a concomitant shift of the N A subtype, which occurred in 1957 ( $\mathrm{H} 2 \mathrm{~N} 2$ replacing $H 1 N 1)$, but not, for example, in 1968, when $\mathrm{H} 3 \mathrm{~N} 2$ replaced $\mathrm{H} 2 \mathrm{~N} 2$ viruses. A shift may occur when an $\mathrm{HA}$-coding segment originating from an avian virus strain replaces the corresponding segment in a human virus to give rise to a virus that can be transmitted between humans [13-15]. A second, fundamentally different mechanism that could give rise to a pandemic is thought to occur when, on rare occasions, an avian virus with an $\mathrm{HA}$ subtype that is different from contemporary human viruses infects humans in toto, and subsequently acquires point mutations that gradually improve its adaptation to the human host until it becomes fully transmissible between individuals $[3,16]$. This mechanism may explain the origin of the Spanish Flu in 1918, but lack of prior virus isolates hampers evidence-based support for this hypothesis. It remains impossible to predict which Influenza A subtype and strain will cause the next pandemic and hence would be a relevant vaccine seed strain. Alternative approaches have been pursued to obtain intra- or even heterosubtypic immunity against influenza by exploiting adjuvant use and more conserved influenza epitopes as novel vaccines $[17,18]$.

$D$ evelopment and use of an ectodomain of matrix protein 2 (M 2e)-based vaccine has several advantages over current influenza vaccines, the most important being the sequence conservation of $\mathrm{M}$ 2e across human Influenza A virus subtypes, which could overcome the need for annual vaccine updates. In addition, it is a membrane protein that is accessible to antibodies, the effectors of most vaccines that are in use. M 2e-based vaccines can be made by recombinant DNA techniques that do not require high biological containment facilities, and their production is possible in economical production systems such as bacteria, permitting scaleup. M 2e-based vaccines are now in Phase I clinical trials.

\section{Biological role of matrix protein 2}

$M$ atrix protein (M ) 2 is a type III membrane protein composed of 97 amino acid residues (aa). It self-assembles as a homotetramer into a protonselective ion channel that is activated by low $\mathrm{pH}$ (Figure 1B) [19]. M 2 consists of a 24-aa ectodomain (M 2e), a 19-aa transmembrane domain and a 54-aa intracellular/intravirion domain. O nly protons can pass through the $\mathrm{M} 2$ channel, by a process that involves protonation of the imidazole group of $\mathrm{H}$ is 37 and that is gate-controlled by Trp41 (Figure 1C) [20]. Following endocytosis of the virus, the influx of protons into the particle facilitates detachment of the ribonucleoprotein complexes from the matrix, an essential step that precedes migration of the genetic material into the cell nucleus. An estimated 10-20 molecules of M 2 protein are present in the virion membrane. H owever, infected cells express M 2 abundantly on internal membranes as well as on the cell surface [21]. M 2 expression in the Golgi apparatus renders the lumen of $G$ olgi vesicles less acidic. This prevents newly expressed $\mathrm{H} A$ from some avian Influenza A strains from adopting its acid-induced fusion state. In particular, this applies to some highly pathogenic avian $\mathrm{H} 5$ or H 7 subtype Influenza $A$ viruses such as $A /$ fowl plague/R ostock/34. H owever, the folding of $\mathrm{HA}$ from most Influenza A virus strains does not seem to depend on the coexpression of $M 2$ [22-25]. The proton channel of $M 2$ is the target of the adamantane family of antivirals, but resistance to these drugs is widespread, mainly owing to single amino acid substitutions in the transmembrane domain of M 2 that abolish drug binding [26-28].

W ith 54 aa, the cytoplasmic domain of $M 2$ is markedly longer than that of $\mathrm{HA}$ (13 residues) and $\mathrm{NA}$ (six residues). C-terminal truncations of the M 2 cytoplasmic domain can decrease its proton channel function [29]. The cytoplasmic tail of M 2 is also involved in virus particle formation and appears to help control virus morphology and packaging of ribonucleoprotein into newly formed virions. This was demonstrated by making use of genetically engineered influenza viruses with a truncated M 2 cytoplasmic 
tail: these mutant viruses produced fewer infectious virus particles compared with wild-type virus. In particular, virus particles generated by infection with a 28-aa deletion mutant virus contained reduced amounts of packaged genome segments [30]. I watsuki-H orimoto et al. confirmed these findings and, in addition, demonstrated that virions obtained from M 2 tail truncated mutant viruses often displayed a filamentous morphology, whereas wild- type virions were spherical [31]. An interaction of the cytoplasmic tail of M 2 with $M 1$ and perhaps the genomic ribonucleoprotein complexes may be essential for proper Influenza A virion formation and budding.

Why is M2e conserved?

$\mathrm{M} 2 \mathrm{e}$ is highly conserved among all known human Influenza A virus strains. Such strong sequence conservation is remarkable for an RN A virus that exists in many subtypes and strains, and it is in sharp contrast with the variability of HA and N A [32], but not with someinternal proteins of the virus, such as the nucleoprotein (NP). A few explanations have been proposed for the limited variation in M 2e sequence:

- Following infection or vaccination with inactivated, licensed influenza vaccines, serum antibody levels specific for $M 2$ e are low in humans, pigs and mouse [33-37]. A presumptive HLA-B27 and HLA-B44 restricted human cytotoxic $\mathrm{T}$-lymphocyte epitope has been identified in $M 2 e$, but its contribution to protection against influenza in the human population, if any, is unknown [38-40]. The adaptive immune response directed to $M 2 e$ is probably limited, which may partially explain its striking sequence conservation across human Influenza A strains.

- There is a genetic constraint on $M 2 e$ variability (Figure 2). The coding sequence of $\mathrm{M} 2 \mathrm{e}$ entirely overlaps that of Influenza A M 1 , which is highly conserved, and so mutations in M 2e are counterselected [21].

- Using domain swapping between M2 and Sendai virusF protein, Park et al. demonstrated that $M$ 2e is needed for $M 2$ incorporation into virions, possibly through an interaction with the membrane proximal part of $\mathrm{HA}$ [41]. Since the $H A 2$ subunit of $H A$ anchors $H A$ in the membrane and, compared with $\mathrm{HAl}$, is rela tively conserved across subtypes, this proposed interaction might also contribute to sequence conservation of $\mathrm{M} 2 \mathrm{e}$ [42].
M2e-specific antibodies protect against Influenza A virus infection

The monoclonal antibody, $14 C 2$, which is directed against M 2e, slows the growth of some virus strains in vitro, but not all [43]. Presumably, this antibody reduces cell surface expression of M 2 and interferes with virion formation in vitro [44]. The first evidence that $14 \mathrm{C} 2$ can also inhibit Influenza A virus replication in vivo was obtained by intraperitoneal injection of the antibody into Balb/c mice, followed by challenge with $\mathrm{A} / \mathrm{U}$ dorn/307/72, an $\mathrm{H} 3 \mathrm{~N} 2$ virus strain that is susceptible to $14 \mathrm{C} 2$ in vitro [45]. This passive immunization resulted in a 100-fold reduction of lung virus titers on days 3 and 4 after challenge compared with mice that received control antibodies.

0 ther researchers developed active vaccination protocols using $M 2$ and mostly M 2e-fusion constructs. Vaccination with an M 2-containing preparation obtained from a recombinant baculovirus insect cell expression system protected mice against challenge with homologous and heterologous Influenza A strains, as evidenced by decreased morbidity and virus replication, without preventing infection [46]. Although serum antibodies reacting specifically with synthetic M 2-derived peptides were present in immunized mice, passive transfer of this M 2 immune serum into naive mice did not confer protection against challenge. This indicates that protection achieved by active vaccination with recombinant M 2 could not be attributed to M 2e-specific antibodies.

The first experimental vaccine to be based solely on $M 2$ e made use of a recombinant viruslike particle (VLP) to display M $2 e$ in a dense, $3 D$, repetitive configuration [47]. Most of the efforts to design M 2e-based vaccines that were reported subsequently also made use of $M 2$ ecarrier conjugates (Table 1). The logic for using VLPS was based on the observation that the highly organized arrangement of natural or artificial epitopes on the surface of VLPs can induce robust B-cell stimulation, most likely by extensive crosslinking of the B-cell receptor [48]. A genetic fusion between $\mathrm{M} 2 \mathrm{e}$ and the hepatitis $\mathrm{B}$ virus core antigen $(\mathrm{HBC})$ was made and expressed in bacteria. When used as a vaccine in mice, M 2e-H Bc VLPs induced a strong antiM 2e specific serum IgG response that protected the animals from a potentially lethal challenge with an HIN 1 or H3N 2 virus; no deaths occurred and morbidity was reduced compared with controls. M 2e- H Bc was also effective when 
Figure 2. Genetic relationship betw een M1 and M2 and comparison of human- and avian-type M2 ectodomain sequences.

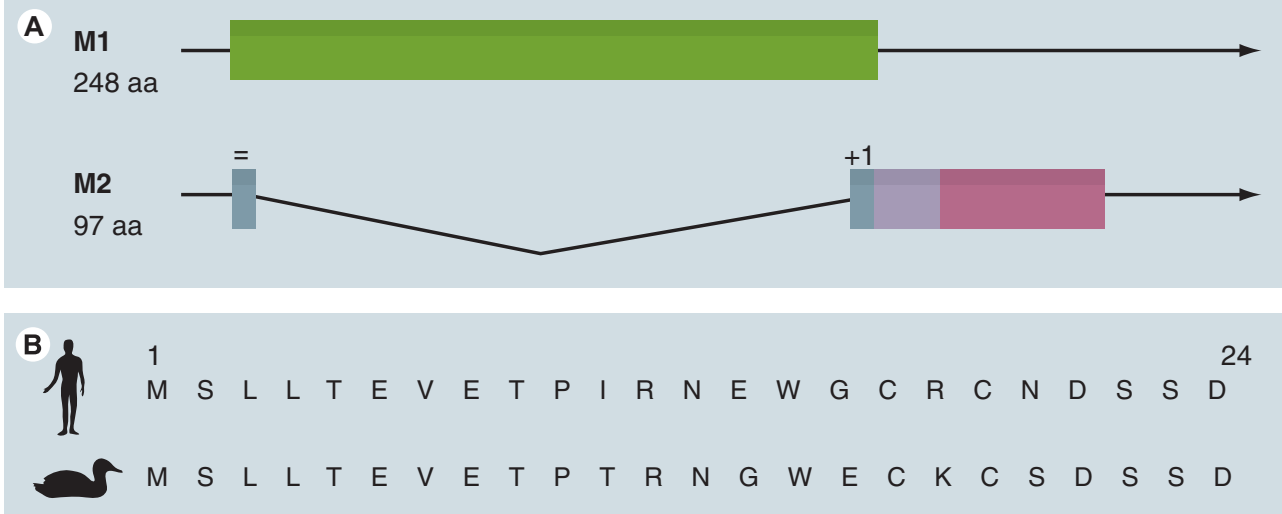

(A) M 2 is translated from a splice variant of the mRNA encoding M 1. The first nine amino acid residues of $M 1$ and $M 2$ are identical (' $=$ ', identical open reading frame). A mino acid residues 10-24 of M2 are translated from the +1 open reading frame $\left({ }^{\prime}+1^{\prime}\right)$ relative to $M 1$. Color codes of the $M 2$ domains are the same as in Figure 1B. (B) The consensus human and avian $M 2$ e sequences differ at five positions. aa: Amino acid residue; $M$ : $M$ atrix protein; $M 2$ e: Ectodomain of $M 2$.

administered intranasally, and protection could be passed to naive mice by passive transfer of $\mathrm{M} 2 \mathrm{e}-\mathrm{HBC}$ immune serum, demonstrating that the humoral anti-M 2 e response was necessary for protection. The $\mathrm{M} 2 \mathrm{e}-\mathrm{H} \mathrm{Bc}$ vaccine has since been improved by making VLPS with three tandem head-to-tail copies of $\mathrm{M}$ 2e. This design improved particle stability and resulted in a tenfold higher M 2e-specific serum IgG titer. By combining the vaccine with adjuvants suitable for humans, a humoral immune response with a balanced IgG 1:IgG 2 a ratio was induced, which is important for protection [49]. More recently, $\mathrm{M} 2 \mathrm{e}-\mathrm{HBC}$ was combined with the mucosal adjuvant CTA1-DD to optimize intranasal administration. This adjuvant was selected because it can promote Th1-directed immune responses without exhibiting toxicity [50-52].

Recombinant M 2 transmembrane deletion constructs were fused to the glutathione-S-transferase coding sequence, and the protein fusions were used to immunize mice. When formulated with incomplete Freund's adjuvant, these proteins induced high M 2e-specific serum antibody titers in mice and reduced lung virus titers following challenge with $\mathrm{H} 3 \mathrm{~N} 2, \mathrm{H} 2 \mathrm{~N} 2$ and $\mathrm{H} 1 \mathrm{~N} 1$ viruses [53]. Jegerlehner $A$ al. used a chemical fusion approach to couple the $M$ 2e peptide to an engineered HBC VLP. Vaccination of mice with this M 2e-VLP conjugate induced a strong IgG response to the peptide and protected animals from a potentially lethal PR8 influenza virus challenge. For unknown reasons, a genetic fusion between $\mathrm{M} 2 \mathrm{e}$ and $\mathrm{HBC}$, similar to the construct that was protective in our study, induced low anti$\mathrm{M} 2 \mathrm{2e}$ serum responses and failed to protect mice from challenge in this study [54]. H owever, we subsequently demonstrated that the chemical fusion construct was not superior to our improved, genetically linked M 2e-H Bc construct [49]. Both chemical and genetic fusions have been exploited by other groups to link $\mathrm{M}$ 2e to various carriers, such as bovine serum albumin, glutathioneS-transferase, keyhole limpet hemocyanin, bacterial outer-membrane complexes, a synthetic, multiple antigen peptide and a 'proprietary hydrophobic domain' (Table 1) [36,37,55-58]. M ost experimental influenza vaccines (including $M 2 e-$ based vaccines), novel adjuvants and fundamental immunological questions related to influenza are first explored in laboratory mice. However, this model clearly has its limitations: M us musculus is not a natural host of Influenza A virus, and most laboratory mice strains used are inbred. A strain of laboratory mice (e.g., Balb/c) typically displays a uniform adaptive immune response following vaccination, and influenza virus challenge strains need to be adapted to the mouse, which involves multiple genetic alterations [59]. Two recent studies demonstrated protection against avian influenza viruses, and the study by Fan et al. includes a vaccination-challenge experiment with $\mathrm{M} 2 e-$ fusion constructs in the ferret, which is considered a natural model for human influenza [56-58]. 
Table 1. Overview of published studies using M2 or M2e vaccine antigens.

\begin{tabular}{|c|c|c|c|c|c|}
\hline M2 antigen & Carrier & Type of fusion & Animal model & Virus & Ref. \\
\hline hM2 & - & - & Mouse & $\mathrm{H} 2 \mathrm{~N} 2, \mathrm{H} 3 \mathrm{~N} 2$ & [46] \\
\hline $\mathrm{hM} 2 \mathrm{e}$ & $\mathrm{HBC}$ & Genetic & Mouse & H1N1, H3N2 & [47] \\
\hline hM 2 deletion constructs & $-/ G S T$ & Genetic & Mouse & $\mathrm{H} 1 \mathrm{~N} 1, \mathrm{H} 2 \mathrm{~N} 2, \mathrm{H} 3 \mathrm{~N} 2$ & [53] \\
\hline hM $2 \mathrm{e}$ & $\mathrm{HBC}, \mathrm{NP}$ & Genetic & Pig & H1N1 & [60] \\
\hline hM2e & $\mathrm{HBC}$ & Genetic, chemical & Mouse & H1N1 & [61] \\
\hline hM 2e & BSA & Chemical & & H3N2 (in vitro) & [36] \\
\hline hM2e & Multiantigen peptide & Chemical & Mouse & H3N2 & [37] \\
\hline hM2e & GST & Genetic & Mouse & H1N1 & [55] \\
\hline $\mathrm{hM} 2 \mathrm{e}$ & $\mathrm{KLH}, \mathrm{OMPC}$ & Chemical & $\begin{array}{l}\text { Mouse, ferret, } \\
\text { rhesus monkey }\end{array}$ & $\begin{array}{l}\text { H1N1, H3N1, } \\
\text { H1N1, none }\end{array}$ & [57] \\
\hline hM 2e & Hydrophobic domain & Genetic & Mouse & $\begin{array}{l}\text { H1N1, H5N1, } \\
\text { H6N2, H9N2 }\end{array}$ & [56] \\
\hline $\begin{array}{l}\text { hM 2e, avM 2e, } \\
\text { M2-DNA vaccine, } \\
\text { M2-adenovirus }\end{array}$ & KLH & Chemical & Mouse & H1N1, H5N1 & [58] \\
\hline
\end{tabular}

Recombinant M2 protein and expression vectors (DNA vaccine or adenovirus) were used as vaccines. M2e vaccine candidates comprise genetic or chemical fusions.

BSA: Bovine serum albumin; HBc: Hepatitis B virus core antigen; GST: Glutathione-S-transferase; hM2: Human type matrix protein 2; hM2e: Human type ectodomain of matrix protein 2; KLH: Keyhole limpet hemocyanin; M: Matrix protein; M2e: Ectodomain of M2; NP: Nucleoprotein; OMPC: Outer membrane protein complex.

O ne group has reported exacerbation of disease following Influenza A virus challenge in pigs that had been immunized intradermally with a DNA construct expressing an M 2e-N P fusion [60]. It is unclear what caused this alleged exacerbation but anti-M 2e specific antibodies per $s e$ were not implicated. Following vaccination and prior to challenge, M 2e-N P gene-vaccinated animals had very low anti-M 2e serum IgG titers $(\sim 1: 70)$ and, as expected, peripheral blood mononuclear cells from these animals proliferated strongly in response to in vitro stimulation with the Influenza A virus strain used for challenge, most likely reflecting NP-specific T-cell responses. Including an N P D N A vaccine control group might have made it possible to draw more specific conclusions on the possible cause of the disease exacerbation. $\mathrm{H}$ einen et al. also evaluated protection in $\mathrm{M} 2 \mathrm{e}-\mathrm{HBC}$-vaccinated pigs. In an adjuvanted $\mathrm{M} 2 \mathrm{e}-\mathrm{H} \mathrm{Bc}$ group, much higher antiM 2e IgG titers ( 1:2000) were obtained and, starting from day 2 after infection, animals in this group had less intense fever compared with a control group. This suggests that anti-M 2e antibodies may provide protection against Influenza A virus infection in pigs, which could be further optimized by using a typical swine influenza $M 2 e$ sequence (six out of 23 residues differed between the $\mathrm{M} 2 \mathrm{e}-\mathrm{HBc}$ vaccine and the swine influenza virus challenge strain used by $\mathrm{H}$ einen et al.).
In conclusion, vaccination with different $\mathrm{M} 2 \mathrm{e}$ fusion constructs can induce protective, humoral immune responses against influenza, resulting in survival and reduced morbidity following a lethal virus challenge.

\section{Mechanism of action}

Anti-M 2e IgG antibodies are instrumental in providing the protection obtained by vaccination with $M 2 e$ vaccines $[37,47,58,61]$, but the mechanism of protection is unclear. $M 2$ in virus particles might be a target of anti-M 2e antibodies. However, virions contain only a few molecules of M2, the external part of which is probably shielded by the much taller $\mathrm{HA}$ and $N A$. This presumably explains why anti-M $2 e$ antibodies fail to neutralize infection in vitro [21,61]. Alternatively, M 2e-specific antibodies could interfere with membrane expression of M 2 and reduce progeny virus yield, as was observed in vitro [44]. This might slow down the infection process at an early stage, which allows the host's innate and adaptive immune compartments to finally control the viral infection. However, the reduction in virus yield by the 14C2 antibody observed in vitro was strain dependent, whereas the efficacy of M 2e-based vaccine candidates has been documented for multiple Influenza A virus subtypes and strains (Table 1). 
Protection induced by M 2e-based vaccines probably depends mostly on the killing of M 2-expressing cells by antibody-dependent cytotoxicity $(A D C C)$. Elimination of infected cells at an early phase of the infection cycle would reduce new virus production, consistent with the reported drop in lung virus titers of M 2e-immune mice. Complement-dependent cytotoxicity appears to play a minor role, because C3-deficient mice are protected against challenge by passive transfer of $M 2$ e-immune serum. By contrast, protection of immunized mice depleted of natural killer (NK) and NK $T$ cells dropped dramatically [61]. Since N K cells mediate $A D C C$ and infiltrate mouse lungs following infection, this study indicates that antiM 2e IgG antibodies and N K cells act in concert to provide protection. By contrast, Thompkins et al. did not observe an effect of NK and N K $\mathrm{T}$-cell depletion on the protection by $\mathrm{M} 2$ vaccination [58]. In that study, M 2 DNA priming, followed by a recombinant $M$ 2-expressing Adenovirus boost, induced protection that was mainly antibody dependent, but $C D 4^{+}$and $C D 8^{+} T$ cells also played a role. These differences in experimental settings and results warrant further research to determine the precise role of the effector cell types (N K, macrophages, or other cells) implicated in anti-M 2e ADCC. Such research may involve the use of mutant mice (e.g., NK deficient) and passive immunization studies using fractionated anti-M 2e IgG isotypes.

M 2e-specific T-cell responses have been reported following vaccination of mice with $\mathrm{M} 2 \mathrm{e}$ constructs, and these responses appear to contribute to protection [37,58]. A concurrent M 2e-specific T helper and cytotoxic T-lymphocyte response may well be advantageous for protection, but probably plays a minor role in the absence of M 2e-specific antibodies. Taken together, anti-M 2e IgG antibodies probably act in concert with effector cells, such as NK and other white blood cells, to induce ADCC of infected M 2-expressing target cells, or with phagocytes that remove infected cells (Figure 3).

Knowledge of the in vivo mechanism of action will be essential for defining correlates of protection (i.e., experimental methods that faithfully reproduce the in vivo processes responsible for the immune protection). H emagglutination inhibition $(\mathrm{HI})$ assays are now routinely used to evaluate efficacy of seasonal influenza vaccines. In this assay, the level of serum IgG antibodies that can block agglutination of red blood cells by influenza virus virions is determined, and the level of the deduced $\mathrm{HI}$ titer correlates well with the degree of protection against the corresponding influenza strain in humans. HI titer, however, is a 'correlate', and there is no evidence that it reflects the true mechanism of protection induced by the vaccination. What quantifiable clinical benefits can one expect from an M 2e-based vaccine given that M 2e-specific immunity is infection permissive? Results obtained in animal models demonstrate that anti-M 2e immunity is associated with reduced viral replication. Hence, a measurable clinical parameter that should be determined in future clinical trials with M 2e-based vaccines is the level of virus shedding in nasal washes of immunized subjects following infection. Ideally, antiM 2e immunity should also be associated with absence of disease symptoms, such as fever. Establishing a well-controlled assay that would allow determination of a true correlate of protection for an M 2e-based vaccine is essential for further clinical development and clearly represents a technical challenge. In vitro quantification of serum ADCC activity may prove to be important for defining correlates of protection. Such an assay could make use of tracker-loaded target cells infected with Influenza A virus or a cell line stably expressing M 2. Introducing M 2eimmune serum together with effector cells (e.g., NK cells enriched from peripheral blood mononuclear cells) would then allow determination of the degree of target cell killing by measuring the release of the uploaded tracker. Alternatively, a fluorescence-activated cell sorting (FACS)-based target cell-killing assay may be developed in which the loss of membrane permeability (a cell death parameter) of fluorescently labeled M 2-expressing target cells would be measured. An analogous assay has been developed to measure specific cytotoxic T -lymphocyte activity [46]. When using infected cells as targets, determining the amount of newly produced virus in antibody-effector cell treated targets could also be considered. H owever, it is clear that such assays are intrinsically more complicated and will require considerable technical development and validation before they can be routinely applied for evaluation of vaccination efficacy.

\section{M2e-escape variants}

$\mathrm{N}$ atural M 2e-specific immune responses are weak, and some concern has been raised that use of an $M 2$ e vaccine could increase immune pressure and promote the evolution of Influenza A 


\section{Figure 3. Putative mechanism of action of anti-M2e antibody-mediated immune protection.}

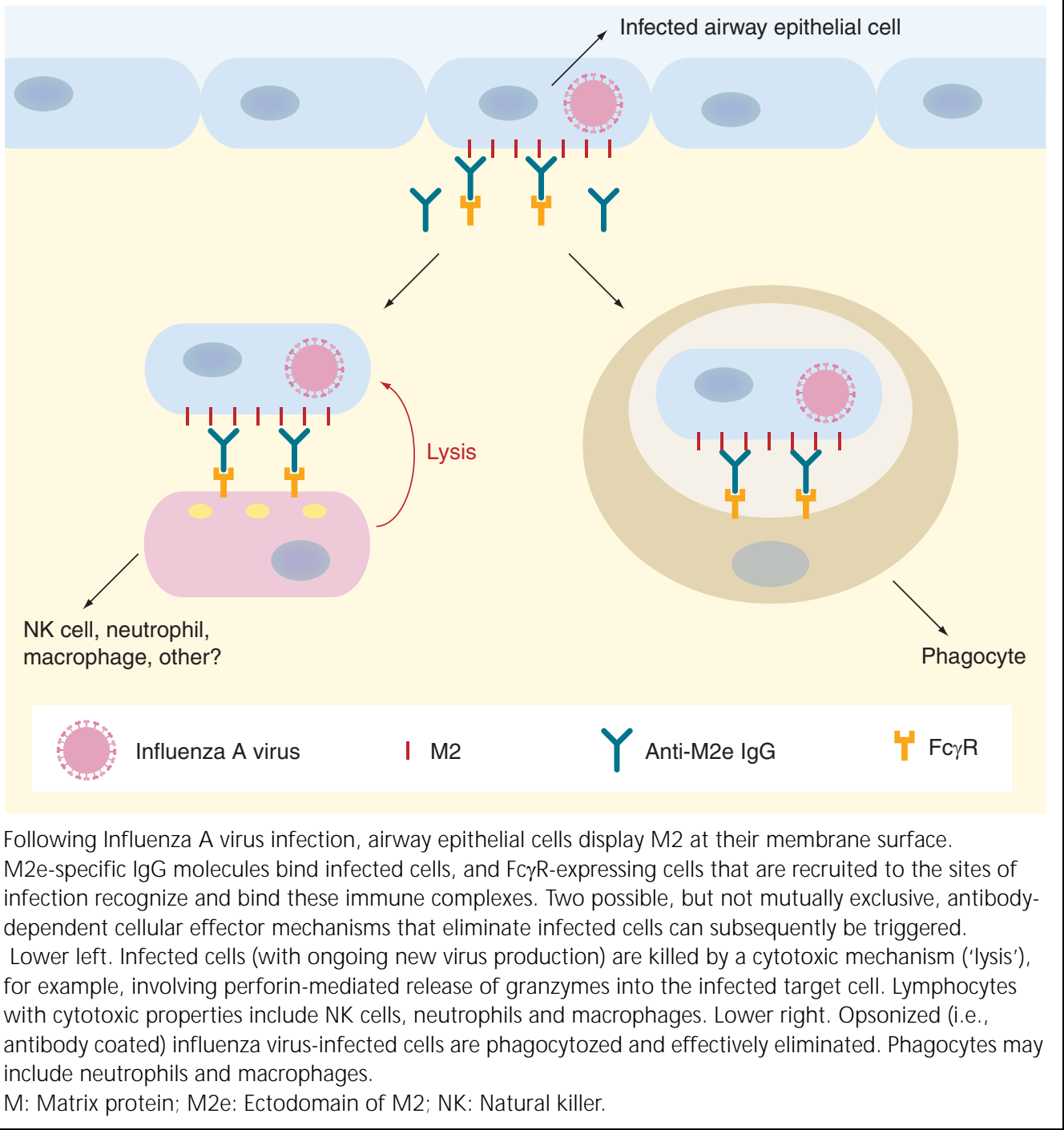

virus escape variants with an altered $\mathrm{M} 2 \mathrm{e}$ sequence. Such escape mutants have been obtained under laboratory conditions. Growth restriction in vitro using the $14 C 2$ monoclonal antibody led to the emergence of an antigenic mutant virus, with a glycine residue replacing glutamic acid at position 8 of M 2e (E8G) [62]. Zharikova et al. used severe combined immunodeficiency (SCID) mice and a slowly progressing infection model using the influenza A PR8 strain to assess the in vivo selection of progeny virus with altered M 2e [63]. Four different M 2e-specific monoclonal IgG 2 a antibodies were used to chronically treat infected mice starting 1 day after infection. Viruses with mutant $\mathrm{M} 2 \mathrm{2e}$ emerged in $65 \%$ of mice but they seemed to be restricted to two M 2e variants: P10L and P1OH. Notably, the
P10L variant was as pathogenic in Balb/c mice as the parental PR8 strain, whereas P10H was less pathogenic. Could M 2e-mutant viruses al so evolve in nature? It is impossible to state with confidence that similar M 2e-escape variants would not evolve through the widespread use of $M$ 2e vaccines, but some observations indicate the improbability of such a scenario. Avian strains, including $\mathrm{H} 5 \mathrm{~N} 1$ and H 9N 2, carrying P1OL or P1OH M 2e exist, but similar human Influenza A viruses have not been isolated. To date, only monoclonal anti-M $2 e$ antibodies have been used to select mutant viruses. However, vaccination with M $2 \mathrm{e}-\mathrm{HBC}$ VLP or homologous $M$ 2efusion constructs induce serum IgG titers in mice that bind strongly to P10L M $2 e$ peptides [D e Filette et al., Unpublished D ata] [58]. Therefore, the induction of an oligoclonal M 2e-specific 
humoral response should be suitable for targeting multiple $\mathrm{M} 2 \mathrm{e}$ variant strains. Furthermore, in $\mathrm{Balb} / \mathrm{c}$ mice, no escape variants could be identified in the presence of antibodies to $M 2 \mathrm{e}[18]$. These mice are immune competent, unlike SCID mice, which lack functional $B$ and $T$ cells, and are deficient in innate immune responses. In an immunecompetent host, vaccination with a suitable $M 2$ efusion construct would induce M 2e-antibodies that may sufficiently thwart infection, ideally without noticeable clinical symptoms. The controlled infection could still induce effective humoral and T-cell responses directed against other antigens of the challenge virus at hand. In this scenario, the virus would be confronted with an immune response targeting multiple epitopes, including $\mathrm{M} 2 \mathrm{e}$, thereby dramatically reducing the chance of escape variant selection.

Single amino acid substitutions in an epitope may prevent a monoclonal antibody from binding, which is reminiscent of many drug-resistance variants that evolve as single amino acid mutations in the drug-targeted site of a protein. Adamantane resistant M 2 variants carry mutations in the transmembrane domain, in which the binding site of this antiviral drug family resides. $\mathrm{H}$ owever, the coding information for this domain does not overlap with the M 1 open reading frame, increasing the theoretical tolerance of mutations in this part of M 2 compared with $\mathrm{M} 2 \mathrm{e}$, for which the coding information entirely overlaps with $M I$ (Figure 2). N evertheless, with or without implementation of an M 2e-based vaccine, worldwide surveillance of influenza virus evolution should be continued in order to catch up with the virus antigenic evolution and to identify antiviral-resistance mutants, and should include monitoring of potential alterations in M 2e.

\section{Conclusion}

$\mathrm{M} 2 \mathrm{e}$ is highly conserved among Influenza A viruses, and its use as a vaccine antigen could greatly simplify influenza vaccine production. Both passive immunization with M 2e-specific monoclonal or polyclonal antibodies and active immunization with different kinds of $\mathrm{M} 2 \mathrm{e}$-carrier constructs can provide intra- and heterosubtypic immunity. Protection is most likely mediated by ADCC directed against infected cells, possibly involving NK cells as well as other effector cells. Under laboratory conditions, M 2e-escape variant viruses have been selected by making use of monoclonal antibodies. As for other infectious diseases, the evolution of the pathogen in the field should be carefully monitored. But, so far, the possibility that a resistant variant influenza strain could emerge under the immune pressure imposed by widespread $M$ 2e vaccination, remains hypothetical. Indeed, several observations argue against this scenario, and any $\mathrm{M} 2 \mathrm{e}$ variations would likely be limited and predictable from the genetic overlap of M 2 with M 1 . C linical studies with an $\mathrm{M} 2 \mathrm{e}$-based vaccine have recently been initiated, and they are expected to reveal its efficacy in a natural Influenza A host setting.

\section{Future perspective}

Because human and avian consensus $M 2 e$ sequences usually differ at no more than five positions, M 2e-based vaccines covering various M 2e sequences could be developed for human use. This approach may broaden immune protection by $\mathrm{M} 2 \mathrm{e}$ even further and embrace the limited M 2e variance that is present in the large pool of subtypes and strains in the natural, mainly avian reservoir of Influenza A viruses. Identifying the immune mechanism of protection by M 2e-specific antibodies will allow identification and validation of correlates of protection. Future efforts will probably include tetrameric $M 2 e$ vaccine constructs to induce antibodies that recognize conformational $\mathrm{M} 2 \mathrm{e}$ epitopes. It is also important to note that influenza B viruses cocirculate with Influenza A viruses in humans and, in some years, are even more prevalent and cause more disease than $\mathrm{A}$ viruses, while displaying an equal amount of antigenic drift. Therefore, a broadly protective human influenza vaccine should include both an Influenza A (e.g., M 2e based) and a B virusderived antigenic component. Influenza $B$ virus encodes two M 2-like molecules: N B and BM 2. Although the ectodomain of $N B(N B e)$ and BM 2 (BM 2e) are both conserved, an approach analogous to M 2e-carrier VLP may be most feasible using N Be (17 aa), since BM 2e comprises only five to seven aas. M 2 e vaccination studies in natural influenza hosts, including ferrets, pigs, birds and humans, are necessary for evaluating the efficacy of this 'universal' Influenza A vaccine approach. Immune protection is not only clinically most relevant in humans but also has to take into account natural challenge doses, transmission and disease progression, which are difficult to mimic in the laboratory. Recently, Acambis Inc., (M A, USA) successfully completed a Phase I vaccine trial with an M $2 \mathrm{e}-\mathrm{H}$ Bc VLP vaccine. It can be safely administered and induces a serum response in 


\section{Executive summary}

\section{Introduction}

- Influenza A and B viruses cause seasonal epidemics that affect 5-10\% of the worlds population each year. M ortality and hospitalization due to influenza occur mainly in the elderly.

- Licensed vaccines are effective in up to $90 \%$ of vaccinated individuals if the vaccines closely match the identity of the emerging epidemic, but they need to be updated each year to keep in pace with the antigenic drift of the influenza virus.

- Influenza A viruses can cause devastating pandemics that are associated with an antigenic shift in the viral hemagglutinin.

\section{Why is the ectodomain of matrix protein 2 conserved?}

- The ectodomain of matrix protein 2 (M 2e) coding region overlaps with that of matrix protein (M)1, and influenza infection is associated with low $\mathrm{M} 2 \mathrm{e}$-specific immune responses.

\section{M2e-specific antibodies protect against Influenza A virus infection}

- Passive immunization of mice with M2e-specific lgG antibodies protects mice against Influenza A virus challenge.

- Current experimental M2e-based vaccines make use of chemical or genetic fusion with a carrier protein.

- Protection by experimental M 2e vaccines has been demonstrated against different virus subtypes, including avian H5N1 and H9N1. Most studies have been performed in mice, but positive results in ferrets and monkeys have also been reported.

\section{Mechanism of action}

- M2e-specific serum IgG antibodies are essential for protection by M2e-based fusion constructs and are the main contributors in M2-based vaccination.

- Anti-M 2e antibodies act in concert with immune effector cells, natural killer cells and likely others to eliminate infected cells by an antibody-dependent cellular cytoxicity mechanism.

\section{Escape variants}

- Viruses encoding an altered M2e sequence have been selected under laboratory conditions in the presence of anti-M2e monoclonal antibodies. It is unlikely that such escape variants would evolve in immune-competent hosts through the use of $M 2 \mathrm{e}$ vaccines that induce an oligoclonal anti-M 2e response.

\section{Future perspective}

- The main unanswered questions regarding M2e-vaccine use are its mechanism of action and its efficacy in humans and other natural hosts.

$90 \%$ of the vaccinees. Future tests will address the efficacy of vaccination-induced $M$ 2e immunity in curtailing Influenza A disease.

\section{Acknowledgements}

S Neirynck provided Figure $1 \mathrm{~A}$ and $\mathrm{M}$ Huyghe produced Table 1.

Financial \& competing interests disclosure Research on influenza in the laboratory of W Fiers and X Saelens is supported by NIH grant 5R01AI055632, Instituut voor de Aanmoediging van Innovatie door Weten- schap en Technologie (IWT), Fonds voor Wetenschappelijk Onderzoek-Vlaanderen (FWO), Ghent University Bijzonder 0 nderzoeksfonds (BOF) and a research collaboration with AcambisInc.

The author has no other relevant affiliations or financial involvement with any organization or entity with a financial interest in or financial conflict with the subject matter or materials discussed in the manuscript apart from those disclosed.

The author thanks Walter Fiers and Amin Bredan for critical reading of the manuscript. N o other writing assistance wasutilized in the production of this manuscript.

\section{Bibliography}

Papers of special note have been highlighted as either of interest $(\bullet)$ or of considerableinterest $(\bullet)$ to readers.

1. Wood JM, Robertson JS: Reference viruses for seasonal and pandemic influenza vaccine preparation. Influenza O ther Respir. Viruses 1(1), 5-9 (2007).

2. M in Jou W, Verhoeyen $M, D$ evos $R$ et al.: C omplete structure of the hemagglutinin gene from the human influenza a/victoria/3/75 (H 3N 2) strain as determined from cloned $D N A$. Cell 19(3), 683-696 (1980).

3. Russell CJ, Webster RG: The genesis of a pandemic influenza virus. Cell 123(3), 368-371 (2005)

-. Very clear review on the genetic mechanisms that explain the evolution of pandemic influenza: reassortment or gradual adaptation to a new host.
4. Verhoeyen M, Fang R, Jou W M et al.: Antigenic drift between the haemagglutinin of the $\mathrm{H}$ ong $\mathrm{K}$ ong influenza strains A/aichi/2/68 and A/victoria/3/75. N ature 286(5775), 771-776 (1980).

5. Smith DJ, Lapedes AS, de Jong JC et al.: $M$ apping the antigenic and genetic evolution of influenza virus. Science 305(5682), 371-376 (2004). 
- Seminal paper illustrating how the use of bioinformatics to evaluate historical serological databases results in a quantitative readout of Influenza A virus antigenic drift. Furthermore, the methodology is now implemented in predicting conventional influenza vaccine strains.

6. Treanor JD : Influenza - the goal of control. N . Engl. J. M ed. 357(14), 1439-1441 (2007).

7. Nichol KL, Treanor JJ: Vaccines for seasonal and pandemic influenza. J. Infect. Dis. 194(Suppl.), 2S111-2S118 (2006).

8. Jefferson T, Rivetti D, Rivetti A et al.: Efficacy and effectiveness of influenza vaccines in elderly people: a systematic review. Lancet 366(9492), 1165-1174 (2005).

9. Jefferson $\mathrm{T}$ : Influenza vaccination: policy versus evidence. Br. M ed. J. 333(7574), 912-915 (2006).

10. Nicholson KG, Wood JM, Zambon M : Influenza. Lancet 362(9397), 1733-1745 (2003).

11. Fouchier RA, M unster $\mathrm{V}$, Wallensten $\mathrm{A}$ et al.: C haracterization of a novel Influenza A virus hemagglutinin subtype (H 16) obtained from black-headed gulls. J. Virol. 79(5), 2814-2822 (2005).

12. Guan $Y$, Poon $L L, C$ heung $C Y$ et al.: H $5 N$ influenza: a protean pandemic threat. Proc. N atl Acad. Sci. U SA 101(21), 8156-8161 (2004).

13. Fang $R, M$ in Jou $W, H$ uylebroeck $D$ et al.: Complete structure of $A /$ duck/ukraine/ 63 influenza hemagglutinin gene: animal virus as progenitor of human $\mathrm{H} 3 \mathrm{H}$ ong $\mathrm{Kong}$ 1968 influenza hemagglutinin. Cell 25(2), 315-323 (1981).

14. Gething MJ, Bye J, Skehel J et al.: Cloning and DN A sequence of double-stranded copies of haemagglutinin genes from $\mathrm{H} 2$ and $\mathrm{H} 3$ strains elucidates antigenic shift and drift in human influenza virus. Nature 287(5780), 301-306 (1980).

15. Scholtissek C, Rohde W, Von H oyningen V et al.: $\mathrm{O} n$ the origin of the human influenza virus subtypes $\mathrm{H} 2 \mathrm{~N} 2$ and $\mathrm{H} 3 \mathrm{~N} 2$. Virology 87(1), 13-20 (1978).

16. Taubenberger JK, Reid AH , Lourens RM et al.: Characterization of the 1918 influenza virus polymerase genes. N ature 437(7060), 889-893 (2005).

17. Leroux-RoelsI, Borkowski A, Vanwolleghem $T$ et al.: Antigen sparing and cross-reactive immunity with an adjuvanted rH 5N 1 prototype pandemic influenza vaccine: a randomised controlled trial. Lancet 370(9587), 580-589 (2007).
18. Gerhard W, M ozdzanowska K, Zharikova D: Prospects for universal influenza virus vaccine. Emerg. Infect. $D$ is. 12(4), 569-574 (2006).

- Review of different strategies, including matrix protein (M )2 targeting, which are being pursued to develop new influenza vaccines aimed at inducing intra- and heterosubtypic immunity.

19. Sakaguchi T, Tu Q, Pinto LH et al.: The active oligomeric state of the minimalistic influenza virus $\mathrm{M} 2$ ion channel is a tetramer. Proc. N atl Acad. Sci. USA 94(10), 5000-5005 (1997).

20. Venkataraman $P, L a m b R A$, Pinto LH : Chemical rescue of histidine selectivity filter mutants of the M 2 ion channel of Influenza A virus. J. Biol. Chem. 280(22), 21463-21472 (2005).

21. Lamb RA, Zebedee SL, Richardson CD : Influenza virus $M 2$ protein is an integral membrane protein expressed on the infected-cell surface. Cell 40(3), 627-633 (1985).

- The first paper that describes the expression of $\mathrm{M} 2$ in influenza-infected cells.

22. Takeuchi K, Lamb RA: Influenza virus M 2 protein ion channel activity stabilizes the native form of fowl plague virus hemagglutinin during intracellular transport. J. Virol. 68(2), 911-919 (1994).

23. Takeuchi K, Shaughnessy M A, Lamb RA: Influenza virus $M 2$ protein ion channel activity is not required to maintain the equine-1 hemagglutinin in its native form in infected cells. Virology 202(2), 1007-1011 (1994).

24. Ciampor F, Bayley PM, N ermut M V et al.: Evidence that the amantadine-induced, M 2-mediated conversion of Influenza A virus hemagglutinin to the low $\mathrm{pH}$ conformation occurs in an acidic trans $\mathrm{G}$ olgi compartment. Virology 188(1), 14-24 (1992).

25. O huchi $M$, Cramer A, Vey $M$ et al.: $R$ escue of vector-expressed fowl plague virus hemagglutinin in biologically active form by acidotropic agents and coexpressed $\mathrm{M} 2$ protein. J. Virol. 68(2), 920-926 (1994).

26. Wang C, Takeuchi K, Pinto LH et al.: Ion channel activity of Influenza A virus M 2 protein: characterization of the amantadine block. J. Virol. 67(9), 5585-5594 (1993).

27. Simonsen L, Viboud C, Grenfell BT et al.: The genesis and spread of reassortment human Influenza $A / H$ 3N 2 viruses conferring adamantane resistance. M ol. Biol. Evol. 24(8), 1811-1820 (2007).
28. Bright RA, M edina M J, Xu X et al.: Incidence of adamantane resistance among Influenza A (H 3N 2) viruses isolated worldwide from 1994 to 2005: a cause for concern. Lancet 366(9492), 1175-1181 (2005).

29. Tobler $K$, Kelly M L, Pinto LH et al.: Effect of cytoplasmic tail truncations on the activity of the $\mathrm{M}(2)$ ion channel of Influenza A virus. J. Virol. 73(12), 9695-9701 (1999).

30. M cC own M F, Pekosz A: The Influenza A virus $M 2$ cytoplasmic tail is required for infectious virus production and efficient genome packaging. J. Virol. 79(6), 3595-3605 (2005).

31. I watsuki-H orimoto $\mathrm{K}, \mathrm{H}$ orimoto $\mathrm{T}, \mathrm{N}$ oda $\mathrm{T}$ et al.: The cytoplasmic tail of the Influenza A virus $\mathrm{M} 2$ protein plays a role in viral assembly. J. Virol. 80(11), 5233-5240 (2006).

32. Sharp PM : O rigins of human virus diversity. Cell 108(3), 305-312 (2002).

33. Black RA, Rota PA, Gorodkova $N$ et al.: Antibody response to the $M 2$ protein of Influenza A virus expressed in insect cells. J. Gen. Virol. 74(Pt 1), 143-146 (1993).

34. Feng J, Zhang $M$, M ozdzanowska $K$ et al.: Influenza $A$ virus infection engenders a poor antibody response against the ectodomain of matrix protein 2. Virol. J. 3, 102 (2006).

35. Kitikoon P, Strait EL, Thacker EL: The antibody responses to swine influenza virus (SIV) recombinant matrix 1 (rM 1), matrix 2 (M 2), and hemagglutinin (H A) proteins in pigs with different SIV exposure. Vet. M icrobiol. 126(1-3), 51-62 (2007).

36. Liu W, Li H, Chen YH : $\mathrm{N}$-terminus of $\mathrm{M} 2$ protein could induce antibodies with inhibitory activity against influenza virus replication. FEM S Immunol. M ed. M icrobiol. 35(2), 141-146 (2003).

37. M ozdzanowska K, Feng J, Eid M et al.: Induction of influenza type A virus-specific resistance by immunization of mice with a synthetic multiple antigenic peptide vaccine that contains ectodomains of matrix protein 2. Vaccine 21(19-20), 2616-2626 (2003).

38. Bui $\mathrm{H} \mathrm{H}$, Peters B, Assarsson E et al.: $A b$ and $T$ cell epitopes of Influenza A virus, knowledge and opportunities. Proc. N atl Acad. Sci. U SA 104(1), 246-251 (2007).

- Comprehensive database covering the known human and mouse B- and T-cell epitopes in Influenza A viruses.

39. Jameson J, Cruz J, Ennis FA: H uman cytotoxic T-lymphocyte repertoire to Influenza A viruses. J. Virol. 72(11), 8682-8689 (1998). 
40. Jameson J, Cruz J, Terajima $M$ et al.: $\mathrm{H}$ uman $\mathrm{CC} 8^{+}$and $\mathrm{CD} 4^{+} \mathrm{T}$ lymphocyte memory to Influenza $A$ viruses of swine and avian species. J. Immunol. 162(12), 7578-7583 (1999).

41. Park EK, C astrucci M R, Portner $A$ et al.: The $M 2$ ectodomain is important for its incorporation into Influenza A virions. J. Virol. 72(3), 2449-2455 (1998),

42. Kovacova A, Ruttkay-N edecky G, $\mathrm{H}$ averlik IK et al.: Sequence similarities and evolutionary relationships of influenza virus A hemagglutinins. Virus Genes 24(1), 57-63 (2002).

43. Zebedee SL, Lamb RA: Influenza A virus M 2 protein: monoclonal antibody restriction of virus growth and detection of M 2 in virions. J. Virol. 62(8), 2762-2772 (1988).

44. H ughey PG, Roberts PC, H olsinger LJ et al.: Effects of antibody to the Influenza $A$ virus $M 2$ protein on $M 2$ surface expression and virus assembly. Virology 212(2), 411-421 (1995).

45. Treanor JJ, Tierney EL, Zebedee SL et al.: Passively transferred monoclonal antibody to the $\mathrm{M} 2$ protein inhibits Influenza $A$ virus replication in mice. J. Virol. 64(3), 1375-1377 (1990).

- First paper to document the growth restriction of Influenza A in vivoby an M 2-specific monoclonal antibody.

46. Slepushkin VA, Katz JM, Black RA et al.: Protection of mice against Influenza $A$ virus challenge by vaccination with baculovirusexpressed M 2 protein. Vaccine 13(15), 1399-1402 (1995).

47. N eirynck S, D eroo T, Saelens $X$ et al.: $A$ universal Influenza $A$ vaccine based on the extracellular domain of the $M 2$ protein. $N$ at. M ed. 5(10), 1157-1163 (1999).

-. First publication demonstrating the protective efficacy of a recombinant protein vaccine based on the conserved M 2 ectodomain fused to a carrier. Protection was demonstrated following both parenteral and mucosal vaccination and shown to be dependent on anti-ectodomain of M 2 (M 2e) IgG antibodies.

48. Noad R, Roy P: Virus-like particles as immunogens. Trends M icrobiol. 11(9), 438-444 (2003).
49. De Filette $M, M$ in Jou W, Birkett $A$ et al.: Universal Influenza A vaccine: optimization of M 2-based constructs. Virology 337(1), 149-161 (2005).

50. Eriksson AM, Schon KM, Lycke N Y: The cholera toxin-derived CTA1-D D vaccine adjuvant administered intranasally does not cause inflammation or accumulate in the nervous tissues. J. Immunol. 173(5), 3310-3319 (2004).

51. De Filette $M$, Fiers $W, M$ artens $W$ et al.: Improved design and intranasal delivery of an $M$ 2e-based human Influenza $A$ vaccine. Vaccine 24(44-46), 6597-6601 (2006).

52. Defilette $M$, Ramne $A$, Birkett $A$ et al.: The universal influenza vaccine $\mathrm{M} 2 \mathrm{e}-\mathrm{H} \mathrm{BC}$ administered intranasally in combination with the adjuvant CTA1-D D provides complete protection. Vaccine 24(5), 544-551 (2006).

53. Frace AM, Klimov Al, Rowe T et al.: M odified M 2 proteins produce heterotypic immunity against Influenza A virus. Vaccine 17(18), 2237-2244 (1999).

54. Jegerlehner $A$, $T$ issot $A$, Lechner $F$ et al.: A molecular assembly system that renders antigens of choice highly repetitive for induction of protective $B$ cell responses. Vaccine 20(25-26), 3104-3112 (2002).

55. Liu W, Peng Z, Liu Z et al.: H igh epitope density in a single recombinant protein molecule of the extracellular domain of Influenza A virus M 2 protein significantly enhances protective immunity. Vaccine 23(3), 366-371 (2004).

56. Ernst WA, Kim HJ, Tumpey TM et al.: Protection against $\mathrm{H} 1, \mathrm{H} 5, \mathrm{H} 6$ and $\mathrm{H} 9$ Influenza $\mathrm{A}$ infection with liposomal matrix 2 epitope vaccines. Vaccine 24(24), 5158-5168 (2006).

57. Fan J, Liang $X, H$ orton $M S$ et al.: Preclinical study of influenza virus A M 2 peptide conjugate vaccines in mice, ferrets, and rhesus monkeys. Vaccine 22(23-24), 2993-3003 (2004)

58. Tompkins SM , Zhao ZS, Lo CY et al.: $M$ atrix protein 2 vaccination and protection against influenza viruses, including subtype H 5N 1. Emerg. Infect. D is. 13(3), 426-435 (2007).

59. Brown EG, Liu H, Kit LC et al.: Pattern of mutation in the genome of Influenza A virus on adaptation to increased virulence in the mouse lung: identification of functional themes. Proc. N atl Acad. Sci. U SA 98(12), 6883-6888 (2001).

60. H einen PP, Rijsewijk FA, de Boer-Luijtze EA et al.: Vaccination of pigs with a D N A construct expressing an influenza virus M 2-nucleoprotein fusion protein exacerbates disease after challenge with Influenza A virus. J. Gen. Virol. 83(Pt 8), 1851-1859 (2002).

61. Jegerlehner A, Schmitz N, Storni T et al.: Influenza $A$ vaccine based on the extracellular domain of $\mathrm{M} 2$ : weak protection mediated via antibody-dependent N K cell activity. J. Immunol. 172(9), 5598-5605 (2004).

- First attempt to clarify the possible mechanism of action of anti-M 2e immunity.

62. Zebedee SL, Lamb RA: Growth restriction of Influenza A virus by M 2 protein antibody is genetically linked to the $M 1$ protein. Proc. Natl Acad. Sci. U SA 86(3), 1061-1065 (1989).

63. Zharikova D, M ozdzanowska K, Feng J et al.: Influenza type A virus escape mutants emerge in vivo in the presence of antibodies to the ectodomain of matrix protein 2 . J. Virol. 79(11), 6644-6654 (2005).

- The emergence of in vivoM 2e-escape virus mutants in the presence of anti-M $2 e$ monoclonal antibodies in an immunocompromised mouse model. M utations appear restricted to one amino acid residue in $M 2 e$.

64. Nishimura K, Kim S, Zhang $L$ et al.: The closed state of a $\mathrm{H}^{+}$channel helical bundle combining precise orientational and distance restraints from solid state N M R. Biochemistry 41(44), 13170-13177 (2002).

Affiliation

- Xavier Saelens

D epartment for M olecular Biomedical Research, VIB, Ghent, Belgium,

and,

D epartment for M olecular Biology,

Ghent U niversity, G hent Technologiepark 927,

B-9052 Ghent, Belgium

Tel.: +32 93313 620;

Fax: +32 93313609 ;

xavier.saelens@dmbr.ugent.be 